\title{
Sex Differences in Cognitive Functioning with Aging in the Netherlands
}

\author{
Astrid C.J. Nooyens ${ }^{a}$ Hanneke A.H. Wijnhoven ${ }^{b}$ Laura S. Schaap ${ }^{b}$ \\ Lena D. Sialino ${ }^{a, b}$ Almar A.L. Kok ${ }^{c}$ Marjolein Visser ${ }^{b}$ \\ W.M. Monique Verschuren ${ }^{a} d \quad H$. Susan J. Picavet ${ }^{a}$ Sandra H. van Oostrom ${ }^{a}$
}

${ }^{a}$ Centre for Nutrition, Prevention and Health Services, National Institute for Public Health and the Environment, Bilthoven, The Netherlands; ${ }^{b}$ Department of Health Sciences, Faculty of Science, Amsterdam Public Health Research Institute, Vrije Universiteit, Amsterdam, The Netherlands; ' Amsterdam UMC, Vrije Universiteit Amsterdam, Department of Epidemiology \& Biostatistics, Amsterdam Public Health, Amsterdam, The Netherlands;

dJulius Center for Health Sciences and Primary Care, University Medical Center Utrecht, Utrecht University, Utrecht, The Netherlands

\section{Keywords}

Aging · Cognitive function · Cohort effect · Longitudinal study $\cdot$ Sex

\begin{abstract}
Introduction: Dementia prevalence in older women is higher than that in men. The purpose of the present study was to investigate whether there is a female disadvantage in cognitive functioning at adult age and/or whether a female disadvantage develops with age. Methods: Data of 5,135 women and 4,756 men from the Longitudinal Aging Study Amsterdam (LASA) and the Doetinchem Cohort Study (DCS) were used. In the LASA, memory, processing speed, fluid intelligence, and global cognitive function were measured every 3-4 years since 1992 in persons aged 55+ years for up to 23 years. In the DCS, memory, processing speed, cognitive flexibility, and global cognitive function were measured every 5 years since 1995 in persons aged 45+ years for up to 20 years. Sex differences in cognitive aging were analyzed using linear mixed models and also examined by the 10-year birth cohort or level of education. Results: Women had a better memory, processing speed, flexibility, and, in the DCS only, global
\end{abstract}

karger@karger.com www.karger.com/ger

Karger!"
C 2022 The Author(s).

Published by S. Karger AG, Basel

This is an Open Access article licensed under the Creative Common Attribution-NonCommercial-4.0 International License (CC BY-NC) (http://www.karger.com/Services/OpenAccessLicense), applicable to the online version of the article only. Usage and distribution for commercial purposes requires written permission. cognitive function than men ( $p^{\prime} s<0.01$ ). However, women showed up to $10 \%$ faster decline in these cognitive domains, except for flexibility, where women showed 9\% slower decline. In the LASA, women scored poorer on fluid intelligence $(p<0.01)$, but their decline was $10 \%$ slower than that in men. Female advantage was larger in later born cohorts; adjustment for the educational level increased the female advantage. Conclusion: Women have better memory and processing speed than men at middle age. This female advantage becomes smaller with aging and has increased in more recent birth cohorts.

(C) 2022 The Author(s).

Published by S. Karger AG, Basel

\section{Introduction}

In 2016, in the Netherlands, the number of women who died with dementia was twice the number of men who died with dementia [1]. Also, the prevalence of dementia is almost twice as high in older women than in men of the same age [2]. Dementia is usually preceded by a long period of decline in cognitive functions [3]. Based on a continuum from healthy cognitive function to de- 
mentia, a lower cognitive function (cognitive reserve) and/or a steeper cognitive decline increases the risk of dementia. The purpose of the present study was to investigate whether there is a female disadvantage in cognitive functioning at adult age and/or whether a female disadvantage develops with age that can explain the sex differences in dementia prevalence at older age.

Sex differences in cognitive function at adult age are well documented: women score higher on cognitive tasks that require verbal processing, while men score higher on tasks that require visuospatial processing [4]. A systematic review showed that sex differences remain similar between the age of $60-80$ years, but that sex differences in cognitive decline may occur after the age of 80 years, although the directions of the associations found were sometimes contradictory [5]. Since this review, some more longitudinal studies showed inconsistent results on sex differences in cognitive decline: either no difference in the rate of cognitive decline between men and women [6], or a steeper decline for men than for women $[7,8]$. The longest follow-up in these studies was 17 years [5-8]. In a recent US study with up to 21 years of follow-up, women had faster declines in global function and executive function, but there was no difference in memory decline between men and women [9]. In this latter study, only few repeated measurements were available for memory. In the present study, we were able to examine cognitive change over a longer period of time (up to 23 years), based on a higher number of repeated cognitive measurements and up to a higher age (100 years), in 2 large Dutch cohort studies. Since secular trends in economic prosperity and gender equity in educational chances may influence sex differences in cognitive function $[4,10]$, we also studied whether sex differences in cognitive functioning differ between different birth cohorts and between different levels of education. We expect that secular trends have improved the average cognitive function of women more than that of men, as the educational opportunities of women have improved in the course of the 20th century due to women's emancipation [11] and education is a key predictor of later life cognitive functioning.

\section{Methods}

We used data from 2 large Dutch aging cohorts: the Longitudinal Aging Study Amsterdam (LASA) [12-14] and the Doetinchem Cohort Study (DCS) $[15,16]$. Both studies have been conducted in line with the Declaration of Helsinki. The LASA has been approved by the Medical Ethics Committee of the VU University medical center and the DCS by the external
Medical Ethics Committee of the Netherlands Organization for Applied Scientific Research and the Medical Ethics Committee of the University of Utrecht. All participants gave written informed consent.

The LASA started in 1992 with 3,107 participants aged 54-85 years from 3 birth cohorts $(1908-1917,1918-1927$, and 19281937). Ten years later, 1,002 persons aged $54-65$ years (born between 1938 and 1947) were added, and in 2012, 1,023 persons aged 54-65 years (born between 1948 and 1957) were added to the ongoing study. Participants are re-measured every 3-4 years. From the LASA, 2,653 women and 2,469 men aged 54-85 years at baseline could be included in our study (see online suppl. Table 1; for all online suppl. material, see www.karger.com/doi/10.1159/520318). The median follow-up was 6 years and ranged from 0 to 23 years (1-8 repeated measurements).

The DCS started in 1987-1991 with an age- and sex-stratified sample of 12,404 men and women aged 20-59 years from the town of Doetinchem. A random selection of 7,769 of these participants was re-invited 6 years later and subsequently has been invited every 5 years. Halfway the second examination round (from 1995 onward), cognitive testing was introduced for participants aged 45 years and over. From the DCS, 2,482 women and 2,287 men aged 45-82 years at baseline, born between 1928 and 1967, were included (see online suppl. Table 2). The median follow-up was 10 years and ranged from 0 to 20 years (1-5 repeated measurements).

\section{Cognitive Function}

In the LASA 4 cognitive tests have been repeatedly carried out: a 15-word learning test [17], a letter substitution task [18], the Raven Colored Progressive Matrices [19] (up to and including the 6th measurement round), and the Mini-Mental State Examination (MMSE) [20]. In the DCS, 4 cognitive tests are repeatedly carried out: a 15-word learning test [21], a letter digit substitution task [22], the Stroop test [23], and a fluency test [24]. Details on these cognitive tests are included as online supplementary material.

\section{Cognitive Domain Scores}

In the LASA, memory function was calculated as the average of the total score on the immediate recalls, the maximum score on the 3 immediate recalls, and the score on the delayed recall of the 15word learning test. Information processing speed was calculated as the average score on the 3 trials of the letter substitution test. Fluid intelligence was calculated as the total score on sections A and B of the Raven Progressive Matrices. Global cognitive function was the maximum score on the MMSE, either based on the subtraction or the spelling backward item.

In the DCS, memory function was calculated as the average of the total score on the immediate recalls, the maximum score on the 3 immediate recalls, and the score on the delayed recall of the 15word learning test. Information processing speed was calculated as the average of the first 2 trials of the Stroop test (reading and color naming) and the score on the letter digit substitution task. Flexibility was calculated as the score on the third trial of the Stroop test (naming ink color). All tests are taken together to calculate a measure for global cognitive function (average z-score on the total of immediate recalls, delayed recall, third trial of the Stroop test (naming ink color), letter digit substitution test, and the fluency test) [25]. 


\section{Demographic Characteristics}

For all participants in both the LASA and DCS, date of birth, age, sex, and the highest attained level of education are ascertained. Based on birth date, participants were categorized in 10-year birth cohorts: 1908-1917, 1918-1927, 1928-1937, 1938-1947, 19481957, or 1958-1967. The highest attained level of education was categorized in 3 levels, based on the distribution in LASA: low (elementary education or less), medium (lower vocational education or general intermediate education), and high (intermediate vocational education, general secondary education, or higher).

\section{Statistics}

In order to compare cognitive function between men and women, reducing the impact of learning effects on cognitive tests, all cognitive data were corrected for the number of cognitive measurements so far, based on the association between the number of measurements (classes) and cognitive function in a linear longitudinal regression model adjusting for age, age $^{2}$, sex, and level of education. After adjusting for the number of measurements, all cognitive data, except for the MMSE scores, were standardized into $\mathrm{z}$-scores based on the mean and standard deviation of the first cognitive measurements of all participants in the study (baseline).

\section{Sex Differences}

To study sex differences in (mean level of) cognitive function, cognitive test scores were included in mixed model analyses with random intercept for the individuals, random slope for age (since this increased model fit significantly, as tested by comparing -2LL between model without and with random slope for age), and an unstructured covariance matrix. Independent variables in these analyses on differences in the level between women and men were sex, age, and age $\mathrm{e}^{2}$ (model 1). If the estimate for sex is statistically significant in this model, positive values denote that women perform better than men do. Negative values denote that men perform better than women do.

Based on this model, difference in cognitive age (expressed in years) was calculated in order to facilitate the interpretation of the differences between men and women at a certain calendar age. At what calendar age is the cognitive function of men at the same level as the cognitive function of women at the age of 65 years? The difference in calendar age is used as an indicator for the difference in cognitive age between men and women (see also online suppl. Fig. 1). So if the cognitive function in women at age 65 years equals the cognitive function of men at age 62 years, the difference in cognitive age is 3 years [26].

To test for sex differences in cognitive decline with aging, the interaction term of sex and age was added. Independent variables in these analyses on differences in cognitive decline with aging were sex, age, age ${ }^{2}$, sex $\times$ age, and sex $\times$ age $^{2}$ (model 2$)$. If an interaction term of sex and age is significant in this model, positive values denote that the decline in cognitive function with aging is stronger in men than that in women. Negative values denote that the decline with aging is stronger in women than that in men. Sex $\times$ age $^{2}$ was only included if this interaction term was significantly associated with cognitive function at $p<0.05$.

Based on these models, difference in cognitive aging was calculated as difference in cognitive decline (in percentage) between men and women between ages 65-75 years. If the interaction term of sex and age was significant in model 2 , this model was used to describe and plot sex differences in cognitive function with aging.
Table 1. Baseline characteristics (participants at their first cognitive measurement)

\begin{tabular}{lll}
\hline & Women & Men \\
\hline LASA & $n=2,654(52 \%)$ & $n=2,469$ \\
Mean age (SD), years & $66.5(8.7)$ & $66.7(8.8)$ \\
$\quad$ Range & $54-88$ & $54-88$ \\
Level of education, \% & & \\
$\quad$ Low* & 39.8 & 25.8 \\
$\quad$ Medium & 32.6 & 31.3 \\
High & 27.6 & 42.9 \\
Birth cohort, \% & & \\
1908-1917 & 21.6 & 23.5 \\
1918-1927 & 19.3 & 18.3 \\
1928-1937 & 19.4 & 19.1 \\
1938-1947 & 19.8 & 19.1 \\
1948-1957 & 19.9 & 20.1 \\
\hline DCS & $n=2,482(52 \%)$ & $n=2,287$ \\
Mean age (SD), years & $55.2(6.6)$ & $55.5(6.6)$ \\
$\quad$ Range & $45-77$ & $45-82$ \\
Level of education, \% & & \\
Low* & 6.7 & 5.7 \\
Medium & 48.2 & 31.9 \\
High & 45.1 & 62.4 \\
Birth cohort, \% & & 13.3 \\
1928-1937 & 17.6 & 31.1 \\
1938-1947 & 27.9 & 13.5 \\
1948-1957 & 37.7 & \\
1958-1967 & 16.9 & \\
\hline
\end{tabular}

Baseline characteristics are given over all first measurements (t0). LASA, Longitudinal Aging Study Amsterdam; DCS, Doetinchem Cohort Study. * Level of education was classified in 3 levels: Low, elementary education or less; Medium, lower vocational education or general intermediate education; High, intermediate vocational education, general secondary education, or higher (higher vocational education/college education/university education).

Otherwise, model 1 was used. If model 2 was used, so decline with aging differed between men and women, it was then examined at which age the sex differences were no longer statistically significantly different.

Associations were considered to be statistically significant at $p<0.05$. Age was centered at 55 years in all analyses on sex differences, in order to present a realistic sex difference (at age 55 years), especially when cognitive decline with aging differs between men and women.

Differences between Birth Cohorts and Levels of Education

Sex differences in level of cognitive function were tested between birth cohorts or between educational levels by adding an interaction term of sex and the subgroups to model 1 (e.g., sex*education). Sex differences in cognitive decline with aging between subgroups were tested by adding an interaction term of sex, age, and the subgroups (3-way interaction: e.g., age* ${ }^{*}$ sed $^{*}$ education) to model 2. Sex differences in cognitive function and cognitive decline with aging within subgroups were calculated in subgroup-stratified analyses. 


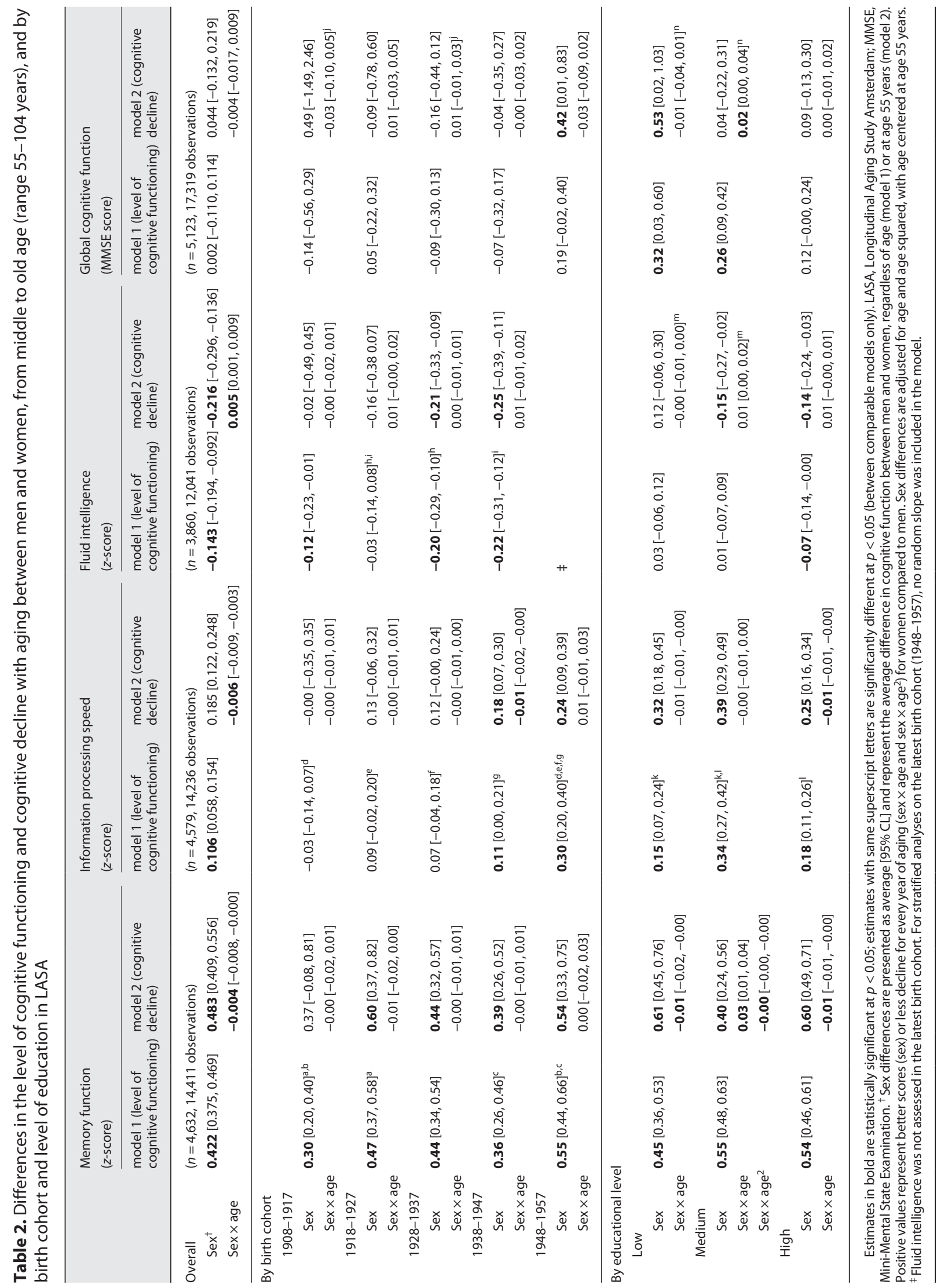


Sensitivity Analyses

In order to test whether menopause influenced results, analyses for DCS were repeated, excluding participants younger than 55 years. Mixed model analyses are able to deal with missing values, based on the assumption that missings are at random. Since missings due to attrition are likely not at random, we repeated our analyses based on data up to age 80 years (LASA and DCS), diminishing attrition not at random due to, e.g., illness and death, and tested whether sex differences were different between participants who did and did not participate for 10 years (LASA) or in the most recent measurement round (DCS).

\section{Results}

In the LASA, the average age at baseline was 66 years and in the DCS, 55 years. In both cohorts, there were about as many men as women. In all birth cohorts, men were higher educated than women and each subsequent birth cohort was higher educated than the previous one (see online suppl. Table 3). More details on demographic characteristics of the study population are presented in Table 1.

\section{Sex Differences in Cognitive Function}

Memory

Women scored better than men on memory in the LASA and DCS (Tables 2, 3). At the age of 65 years, women were cognitively $>10$ years younger than men. This female advantage became smaller with aging due to stronger decline in women ( $9 \%$ between ages $65-75$ years). Despite this convergence, the sex difference in memory remained statistically significant until after the age of 85-90 years (see also Fig. 1).

\section{Information Processing Speed}

In the LASA and DCS, women scored better than men on information processing speed (Tables 2,3). At the age of 65 years, women were cognitively 3 years younger than men. This female advantage became smaller with aging due to stronger decline in women $(7-10 \%$ between ages 65-75 years). Due to this convergence, the sex difference in processing speed lost its statistical significance between the ages of 75-80 years (see also Fig. 1).

\section{Fluid Intelligence}

Women scored lower than men on fluid intelligence (Table 2). At the age of 65 years, women were cognitively 4 years older than men. This female disadvantage became smaller with aging due to smaller decline in women $(10 \%$ between ages 65 and 75 years). The sex difference in fluid intelligence lost its statistical significance between the age of $80-85$ years (see also Fig. 1).

Sex Differences in Cognitive Aging
Cognitive Flexibility

Women scored better than men on cognitive flexibility (Table 3). At the age of 65 years, women were cognitively 3 years younger than men. This female advantage further increased with aging due to smaller decline in women (9\% between ages 65 and 75 years, see also Fig. 1).

\section{Global Cognitive Function}

In the LASA, no significant differences in global cognitive function or decline in global cognitive function (based on MMSE) with aging were observed between men and women (Table 2; Fig. 1). In the DCS, women scored better than men on global cognitive function (based on all cognitive tests, Table 3). At the age of 65 years, women were cognitively 4 years younger than men. This female advantage became smaller with aging due to stronger decline in women ( $10 \%$ between ages 65 and 75 years). Despite this convergence, the sex difference in global cognitive function in the DCS remained statistically significant until after the age of 85 years (see also Fig. 1).

\section{Sex Differences by Birth Cohorts}

Female advantage was larger in the most recent birth cohort than the earlier birth cohorts for memory and information processing speed (Tables 2, 3), and global cognitive function (in DCS only, Table 3). In analyses stratified for birth cohort, cognitive decline was not consistently different between men and women, or between birth cohorts.

\section{Sex Differences by Educational Levels}

Sex differences in the level of cognitive function were not statistically different between levels of education, except for information processing speed in LASA (Tables 2, 3 ). For processing speed, the female advantage was significantly larger in the medium educated participants than in the low- and high-educated groups. The female advantage in cognitive function within different levels of education appeared larger than the overall female advantage. Moreover, sex differences in fluid intelligence were no longer present, while for global cognitive function in the LASA, a female advantage appeared in stratified analyses. Also, for fluid intelligence, cognitive flexibility, and global cognitive function (LASA only), women in the medium educated group showed less cognitive decline than those in the low-educated group (Tables 2, 3).

\section{Sensitivity Analyses}

Analyses excluding participants younger than 55 years showed similar or smaller estimates for overall sex differ- 
LASA
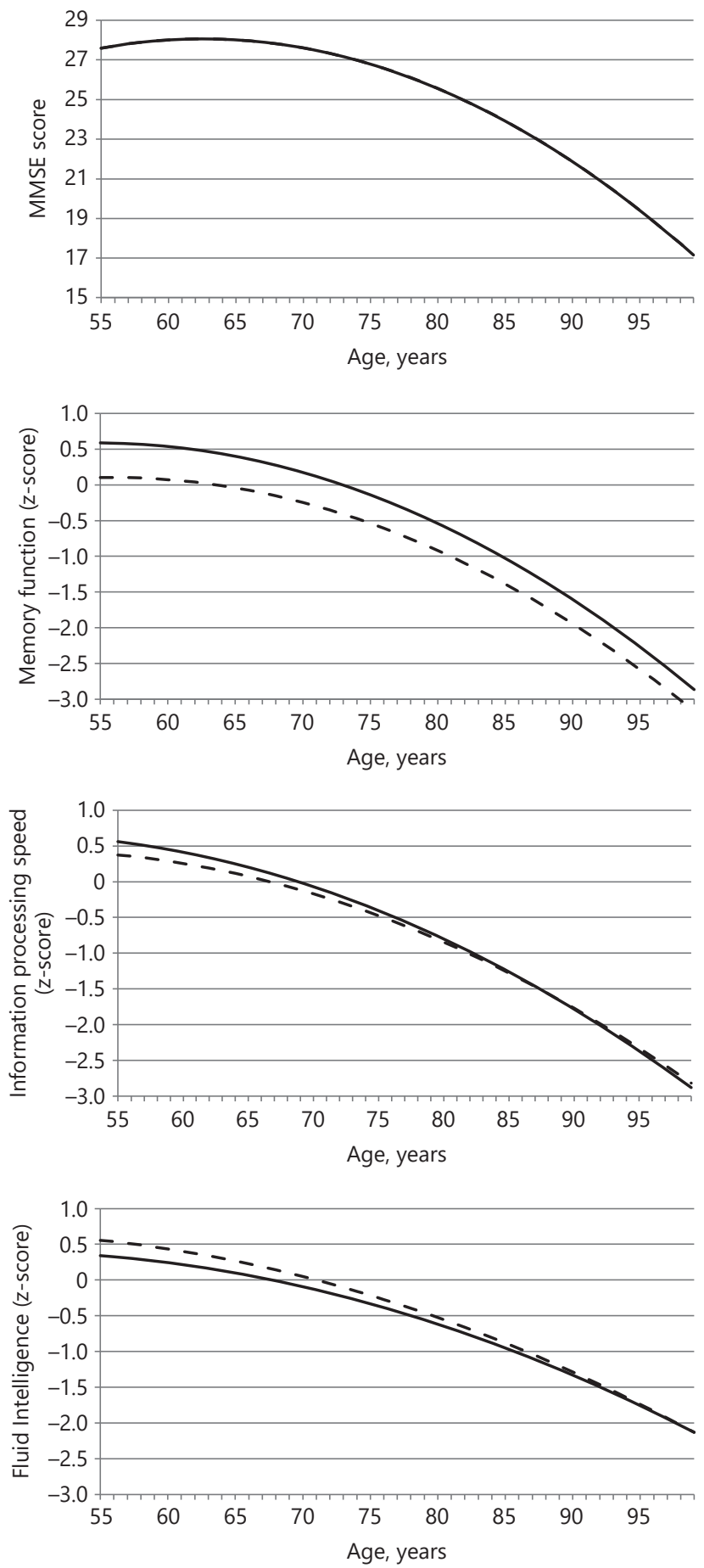

DCS
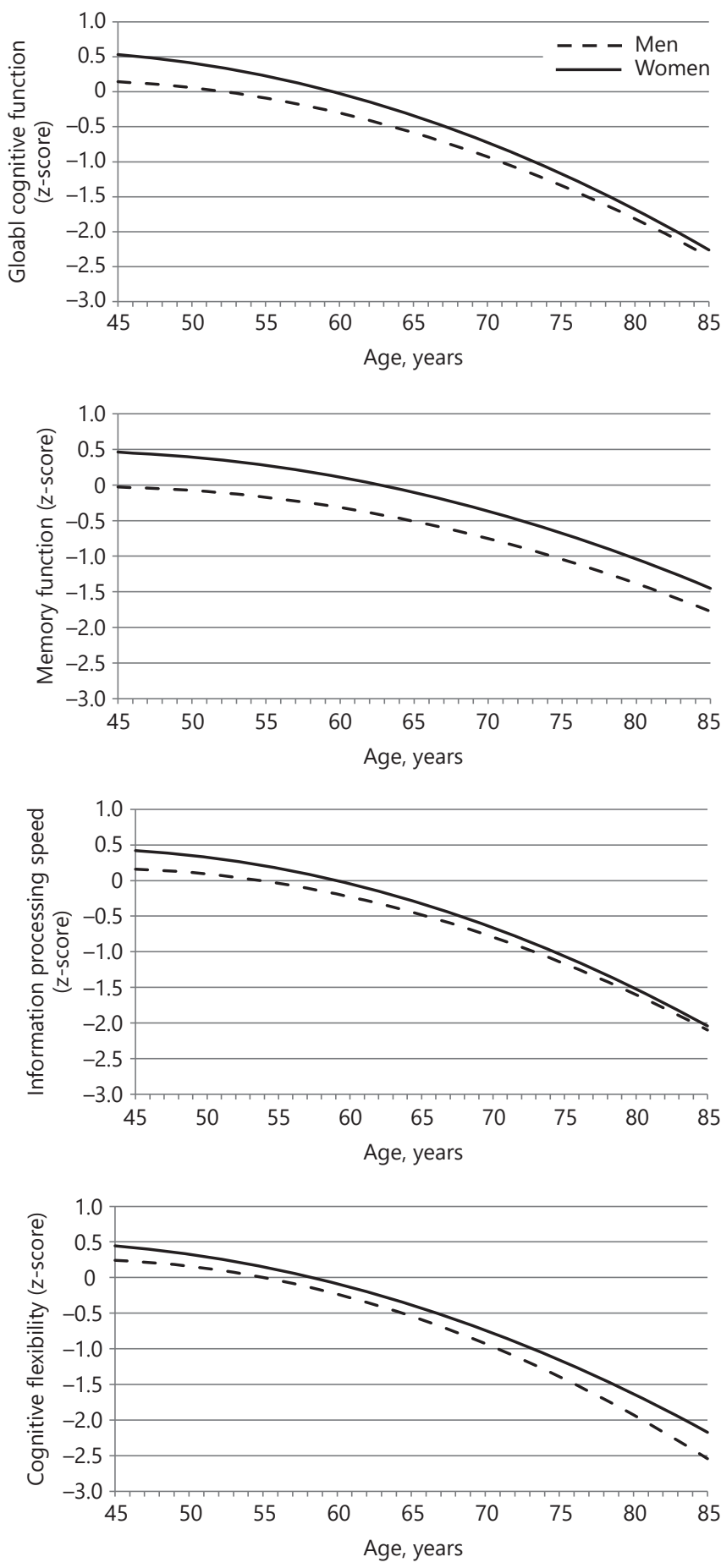

Fig. 1. Cognitive functions with aging for men and women in the LASA (plotted for age 55-99 years) and DCS (plotted for age 45-85 years). Note that MMSE scores with aging of men and women in LASA are similar, and therefore, only 1 line is visible in the figure. LASA, Longitudinal Aging Study Amsterdam; DCS, Doetinchem Cohort Study; MMSE, Mini-Mental State Examination. 


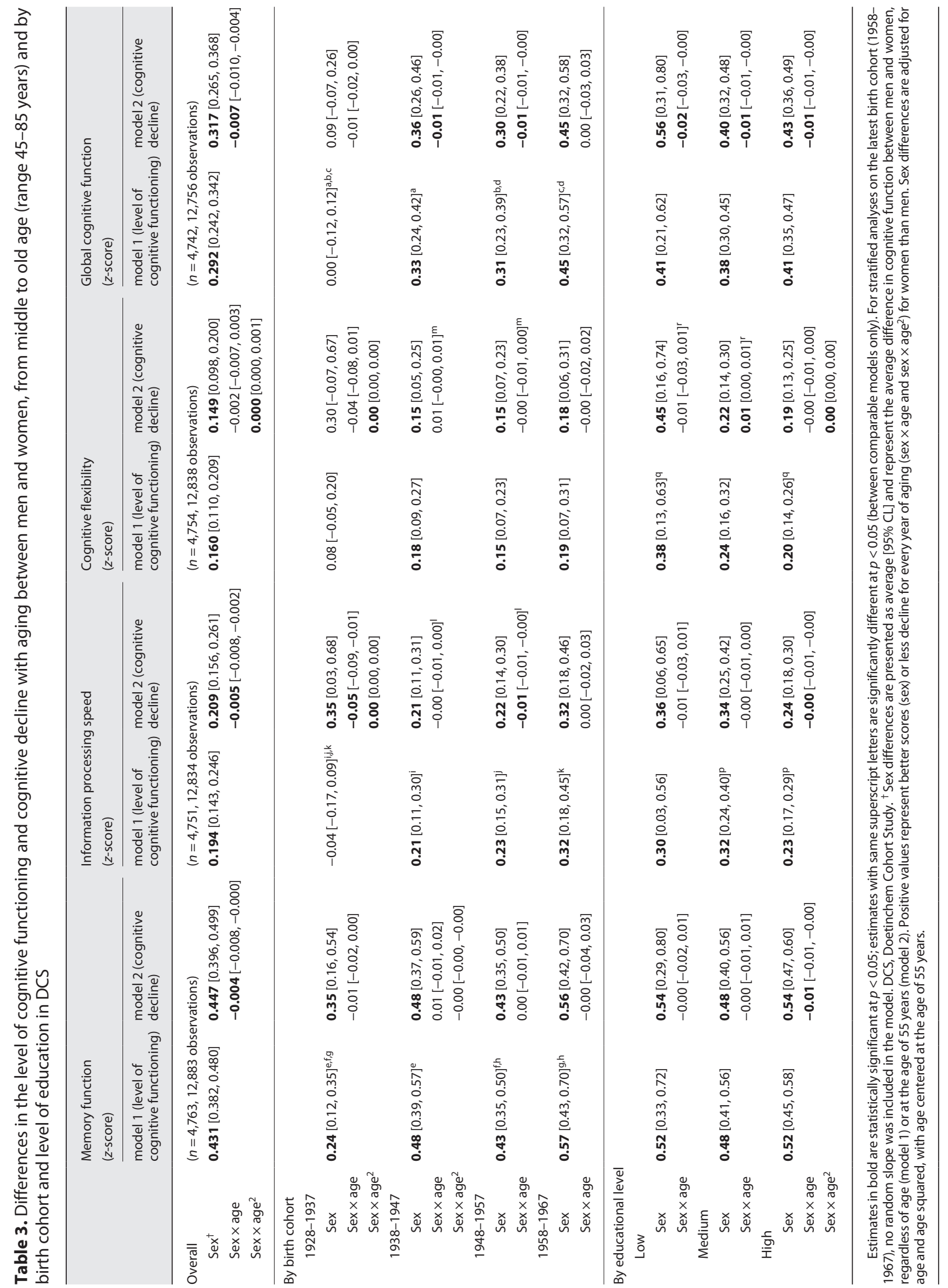


ences in cognitive function, but sex differences in cognitive change were larger for memory, and decline in flexibility was now significantly larger in men than that in women (online suppl. Table 6). Analyses on data from participants up to 80 years old yielded comparable results to those based on all available data (online suppl. Table 7). In the LASA, sex differences in memory function, but not memory decline, were larger in the group who remained $>10$ years in the study than persons who dropped out earlier. In the DCS, although persons who did not participate in the most recent measurement round had worse cognitive function and declined faster, attrition did not affect sex differences in cognitive function or cognitive decline.

\section{Discussion}

The objective of this study was to investigate sex differences in cognitive function at middle age and in cognitive decline with aging from middle to old age. We observed a female advantage for memory function, information processing speed, and cognitive flexibility; a female disadvantage for fluid intelligence; and no sex differences (in LASA) or a female advantage (in DCS) for global cognitive function. Overall, these sex differences in cognitive function declined with aging, since women showed faster cognitive decline in memory and information processing speed than men. Sex differences in cognitive function were larger in more recent birth cohorts. After adjustment for the educational level, the female advantages were larger, indicating that, particularly in earlier cohorts, the lower average education level of women suppressed part of the sex differences in cognitive functioning.

In our study, men outperformed women in spatial tests (fluid intelligence) and women outperformed men on verbal tests (memory test) and speed tests (substitution test). In an opinion article, Hyde [4] wrote that such differences between men and women existed in psychological research from the 1930s through the 1970s, but that these phenomena may have narrowed over time [4]. In our longitudinal datasets, however, we observed that both the female advantages (memory and information processing speed) as well as the female disadvantage (fluid intelligence) became larger in later birth cohorts. The larger female advantages in more recent birth cohorts may be explained by the fact that inequalities in educational attainment between men and women declined across birth cohorts. However, the increase of the female disadvantage on fluid intelligence with more recent birth cohort is not easily explained. Based on our results, existing differences between men and women seem to have enlarged over time.

In agreement with the systematic review [5], earlier analyses on sex differences in cognitive decline over, respectively, 6 and 16 years of follow-up in the LASA revealed no significant sex differences $[27,28]$. However, in the present study with 23 years of follow-up, we did observe sex differences in cognitive decline in the LASA. The disagreement may be explained by the lack of sex differences in cognitive decline in earlier birth cohorts in the LASA (Table 2). Later studies observed either no sex differences in cognitive decline [6], or, in contrast to our results, observed less cognitive decline among women [7, 8]. For instance, in the English Longitudinal Study of Ageing [8], women aged 50 years and over showed significantly less decline in memory and global cognitive function than men over 8-year period of follow-up. One recent study among US adults [9] showed faster cognitive decline in women for global cognitive function and executive function, but not for memory. In both our cohorts, cognitive decline in memory function as well as processing speed was faster in women than in men. The differences in results can possibly be explained by the fact that we have more repeated measurements, and thus more power, for memory than in Levine's study.

Memory is typically first affected in Alzheimer's disease, while processing speed is, among other domains, affected in vascular dementia. Therefore, the stronger decline in these domains in women may indicate higher risk of these types of dementia at older age in women. We did not investigate sex differences in different types of dementia, but questioned whether sex differences in cognitive function and/or cognitive decline at adult age exist. These may explain sex differences in the total prevalence of dementia, based on the assumption that lower cognitive reserve and/or stronger cognitive decline in general increases the risk of dementia at older age.

In our analyses, with up to 23 years of follow-up, we observed that, in general, sex differences in cognitive functioning became smaller from middle into old age. Cognitive function was modeled as a (nonlinear) function of age, since age is the most important determinant of cognitive functioning [5]. Sex significantly interacted with age on cognitive functioning in the overall analyses. In the stratified analyses, this interaction was less clear and significant in only few birth cohorts (Tables 2,3 ). It was not clear whether the sex differences in cognitive decline in the overall models actually were artifacts based on 
differences in sex differences between birth cohorts (earlier cohorts showing smaller sex differences in level of cognitive function than later born cohorts). Therefore, ad hoc analyses were performed with birth cohort and educational level as covariates in the models. Now, again, significant interactions of sex and age on cognitive functioning were observed (online suppl. Tables 4,5 ), indicating that the sex differences in cognitive decline in our population were not only artifacts but also present within birth cohorts.

Although women outperformed men on memory function and information processing speed, their cognitive functions declined stronger with aging. Among the older population of Cache Country (USA), the incidence of $\mathrm{AD}$ in women was similar before the age of 85 years, but greater than men after the age of 85 years [29]. In European populations, the prevalence of dementia in men and women start to diverge between the age of 80 and 85 years, to the detriment of women [30]. This age range is about equal to the age range where cognitive functions of men and women start to cross in our analyses (information processing speed and [in DCS] global cognitive function). This disappearance of the female advantage may partly explain the female disadvantage in dementia incidence at very old age.

The difference in cognitive functions between men and women became smaller at higher age, since cognitive decline was larger in women than in men. It was not in the scope of the present study to elucidate on determinants of these differences. Several mechanisms have been postulated to explain sex differences in cognitive decline and/or dementia prevalence: first, estrogen loss in postmenopausal women may explain a stronger cognitive decline with aging, especially in verbal tasks [31]. Typically, we observed a higher rate of cognitive decline in women for memory and processing speed (and the sex differences in these declines were larger after the age of 55 years in DCS), but not for flexibility and fluid intelligence. Therefore, estrogen loss may partly explain the observed sex differences in cognitive decline in the present study. However, one may speculate that women in more recent birth cohort may more often use estrogens as hormone therapy around menopause, but sex differences in cognitive decline between birth cohorts did not reflect such a secular trend. Second, a greater cognitive reserve in men could explain higher dementia prevalence at similar age in women [31]. Individuals with greater cognitive reserve, as reflected in years of education, are better able to cope with $\mathrm{AD}$ brain pathology without observable deficits in cognition [32]. This may have been the case in our study.
Especially in earlier birth cohorts, women are lower educated, and may therefore have lower cognitive reserve than men. However, women score higher on most cognitive tests, which is not in line with this explanation. Third, selective survival or competing risk could explain sex differences in cognitive decline. Based on the results in the Framingham study [33], it is questioned whether the higher lifetime risk of dementia for women actually is a female disadvantage. An important competing risk for cognitive decline should not be eliminated, namely, mortality. Men have a lower life expectancy than women because they die younger due to stroke or other cardiovascular diseases. Therefore, it can also be argued that women have a higher risk of dementia due to a lower risk to die from other diseases earlier in life. This hypothesis was verified in more recent analyses on the Framingham study that concluded that selective survival of men with a healthier cardiovascular risk profile and hence lower propensity to dementia might partly explain the higher lifetime risk of dementia/Alzheimer's disease in women [34]. Also in our study, the percentage of (surviving) women increased with longer follow-up, especially at higher ages in the LASA. Our analyses excluding participants over 80 years generated similar, although somewhat larger sex differences in cognitive aging, indicating that healthy survivors narrow the sex differences in cognitive aging. Probably, especially more healthy men with better cognitive health survive and thereby close the cognitive gap between men and women at higher age, when less healthy men have dropped out due to illness or death. Other explanations for sex differences in cognitive decline may be found in differences in lifestyle or differences in cardiovascular risk factors. Another reason could also be a statistical one: since women scored higher at baseline, they have a higher chance to decline stronger over time than men (regression to the mean).

The LASA and DCS are both large population-based cohort studies with a long follow-up period for cognitive function. Therefore, these cohorts provide data over a large age range over different birth cohorts. Sensitive cognitive test batteries produced large ranges of cognitive performance, already from middle age. As all (long-lasting) cohort studies, both the LASA and DCS suffer from (selective) attrition, resulting in relatively healthy cohorts. Nevertheless, also these healthy cohorts showed clear cognitive decline with aging, as well as differences between men and women, birth cohorts, and educational levels. Persons who dropped out had on average lower memory function than persons who did not drop out, but differences between men and women in memory func- 
tion and memory decline were not different in the DCS (data not shown). In the LASA, sex differences in memory function, but not memory decline, were larger in the group who remained $>10$ years in the study than in persons who dropped out earlier. This means that sex differences observed in the LASA may be overestimated due to selective attrition. However, based on our sensitivity analyses on attrition in the DCS, attrition did not affect estimates on sex differences. Since sex differences in the LASA and DCS were comparable, we believe a potential overestimation in LASA was only marginal. Another point is the large difference in the educational level between the DCS and LASA. In the early 20th century, girls, regardless of their cognitive abilities, often did not attend school longer than legally required: up to and including primary school, as was especially seen in our earlier cohorts. With women's emancipation, it also became more common for girls to attend school longer and the level of schooling better matches their cognitive abilities [11]. This trend was confirmed in our population (online suppl. Table 3). In both our cohorts, both the decline in percentage of low-educated participants and the increase in percentage of high-educated participants were larger in women than in men. Thus, as a proxy for cognition, education is better suited in later cohorts than in earlier ones.

In conclusion, at middle age, women had a better cognitive function than men, but most sex differences in cognitive function narrowed with aging. The observation that women decline faster in memory and information processing speed may reflect the sex differences in dementia prevalence as observed at old age. Female advantage was larger in more recent birth cohorts. This may have implications for future sex differences in dementia incidence and prevalence.

\section{Acknowledgments}

The authors thank the respondents, epidemiologists, and fieldworkers of the Longitudinal Aging Study Amsterdam and the $\mathrm{Mu}-$ nicipal Health Service in Doetinchem for their contribution to the data collection for this study.

\section{Statement of Ethics}

Both the DCS and LASA have been conducted in line with the Helsinki Declaration. The LASA has been approved by the Medical Ethics Committee of the VU University medical center (reference numbers 92/138, 2002/141 and 2012/361) and the DCS by the external Medical Ethics Committee of the Netherlands Organization for Applied Scientific Research (MEC codes 93/01 and 98/01) and the Medical Ethics Committee of the University of Utrecht (WMO protocol number 07/233 and METC protocol number 07-233/O). All participants gave written informed consent.

\section{Conflict of Interest Statement}

None of the authors reported a potential conflict of interest.

\section{Funding Sources}

This work was supported by the Netherlands Organization for Health Research and Development (ZonMw 849200005). The Longitudinal Aging Study Amsterdam is supported by a grant from the Netherlands Ministry of Health, Welfare, and Sport, Directorate of Long-Term Care. The data collection (in 2012-2013 and 2,013-2,014) was financially supported by the Netherlands Organization for Scientific Research (NWO) in the framework of the project "New Cohorts of young old in the 21st century" (file number 480-10-014). The Doetinchem Cohort Study is financially supported by the Dutch Ministry of Health, Welfare, and Sport, and the National Institute for Public Health and the Environment. The data up to and including 1997 were additionally financially supported by the Europe against Cancer program of the European Commission (DG SANCO). The funders had no role in the design of the study, the collection and analysis of data, and the decision to publish or the preparation of the manuscript.

\section{Author Contributions}

All authors met the criteria for authorship of the International Committee of Medical Journal Editors. Specific contributions: S.H.O., L.S.S., and H.A.H.W. contributed to conception and acquisition of the work; A.C.J.N. contributed to design, analysis, and drafting; and all authors contributed to interpretation of data, revising, and final approval of the manuscript.

\section{Data Availability Statement}

The data of the Doetinchem Cohort Study pertaining to this manuscript are available upon request. We are unable to place data in a public repository due to legal and ethical constraints. The participants' informed consent did not include consent to public availability of the data. However, the data are available upon request. We advise researchers to contact the corresponding author, who will contact the scientific committee of the Doetinchem Cohort Study. 


\section{References}

1 CBS. One in ten deaths attributed to dementia: Statistics Netherlands (CBS); 2017 [updated 2017 Jul 19]. Available from: https:// www.cbs.nl/en-gb/news/2017/29/one-inten-deaths-attributed-to-dementia.

2 Prince MJ, Wimo A, Guerchet M, Ali G-C, Wu Y-T, Prina M. World Alzheimer report 2015. 2015.

3 Rajan KB, Wilson RS, Weuve J, Barnes LL, Evans DA. Cognitive impairment 18 years before clinical diagnosis of Alzheimer disease dementia. Neurology. 2015;85(10):898-904.

4 Hyde JS. Sex and cognition: gender and cognitive functions. Curr Opin Neurobiol. 2016; 38:53-6.

5 Ferreira L, Ferreira Santos-Galduróz R, Ferri $\mathrm{CP}$, Fernandes Galduróz JC. Rate of cognitive decline in relation to sex after 60 years-of-age: a systematic review. Geriatr Gerontol Int. 2014;14(1):23-31.

6 Caselli RJ, Dueck AC, Locke DE, Baxter LC, Woodruff BK, Geda YE. Sex-based memory advantages and cognitive aging: a challenge to the cognitive reserve construct? J Int Neuropsychol Soc. 2015;21(2):95-104.

7 McCarrey AC, An Y, Kitner-Triolo MH, Ferrucci L, Resnick SM. Sex differences in cognitive trajectories in clinically normal older adults. Psychol Aging. 2016;31(2):166-75.

8 Zaninotto P, Batty GD, Allerhand M, Deary IJ. Cognitive function trajectories and their determinants in older people: 8 years of follow-up in the English Longitudinal Study of Ageing. J Epidemiol Community Health. 2018;72(8):685-94.

9 Levine DA, Gross AL, Briceno EM, Tilton N, Giordani BJ, Sussman JB, et al. Sex differences in cognitive decline among US adults. JAMA Netw Open. 2021;4(2):e210169.

10 Miller DI, Halpern DF. The new science of cognitive sex differences. Trends Cogn Sci. 2014;18(1):37-45.

11 Vermeulen M, Waslander S. The Dutch way in education. Chapter 6: excellence in emancipation, a century-long search for balance. Helmond, The Netherlands: Uitgeverij OMJS; 2017.

12 Huisman M, Poppelaars J, van der Horst M, Beekman AT, Brug J, van Tilburg TG, et al. Cohort profile: the Longitudinal Aging Study Amsterdam. Int J Epidemiol. 2011;40(4):86876.

13 Hoogendijk EO, Deeg DJ, Poppelaars J, van der Horst M, Broese van Groenou MI, Comijs HC, et al. The Longitudinal Aging Study
Amsterdam: cohort update 2016 and major findings. Eur J Epidemiol. 2016;31(9):927-45.

14 Hoogendijk EO, Deeg DJH, de Breij S, Klokgieters SS, Kok AAL, Stringa N, et al. The Longitudinal Aging Study Amsterdam: cohort update 2019 and additional data collections. Eur J Epidemiol. 2019;35:61-74.

15 Verschuren WM, Blokstra A, Picavet HS Smit HA. Cohort profile: the Doetinchem Cohort Study. Int J Epidemiol. 2008;37(6):123641.

16 Picavet HSJ, Blokstra A, Spijkerman AMW, Verschuren WMM. Cohort profile update: the Doetinchem Cohort Study 1987-2017: lifestyle, health and chronic diseases in a life course and ageing perspective. Int J Epidemiol. 2017;46(6):1751-g.

17 Saan RJ, Deelman BG. Nieuwe 15-woorden test A en B (15WTA en 15WTB) [new version of 15 words test (15WTA and 15WTB)]. In: Bauma AMJ, Lindeboom J, editors. Neuropsychologische diagnostiek Handboek [Neuro-psychological diagnostics handbook]. Amsterdam: Swets \& Zeitlinger; 1986. p. 1328.

18 Piccinin AM, Rabbitt PM. Contribution of cognitive abilities to performance and improvement on a substitution coding task. Psychol Aging. 1999;14(4):539-51.

19 Raven J, Raven JC, Court JH. Raven manual: selection 1. General overview. Oxford: Psychologist Press; 1995.

20 Folstein MF, Folstein SE, McHugh PR. "Minimental state". A practical method for grading the cognitive state of patients for the clinician. J Psychiatr Res. 1975;12(3):189-98.

21 Van der Elst W, van Boxtel MP, van Breukelen GJ, Jolles J. Rey's verbal learning test: normative data for 1855 healthy participants aged 24-81 years and the influence of age, sex, education, and mode of presentation. J Int Neuropsychol Soc. 2005;11(3):290-302.

22 Van der Elst W, van Boxtel MP, van Breukelen GJ, Jolles J. The letter digit substitution test: normative data for 1,858 healthy participants aged 24-81 from the Maastricht Aging Study (MAAS)-influence of age, education, and sex. J Clin Exp Neuropsychol. 2006;28(6): 998-1009.

23 Van der Elst W, van Boxtel MP, van Breukelen GJ, Jolles J. The Stroop color-word test: influence of age, sex, and education; and normative data for a large sample across the adult age range. Assessment. 2006;13(1):6279.
24 Van der Elst W, van Boxtel MP, van Breukelen GJ, Jolles J. Normative data for the animal, profession and letter $M$ naming verbal fluency tests for Dutch speaking participants and the effects of age, education, and sex. J Int Neuropsychol Soc. 2006;12(1):80-9.

25 Nooyens AC, Bueno-de-Mesquita HB, van Boxtel MP, van Gelder BM, Verhagen H, Verschuren WM. Fruit and vegetable intake and cognitive decline in middle-aged men and women: the Doetinchem Cohort Study. Br J Nutr. 2011;106(5):752-61.

26 Nooyens ACJ, Yildiz B, Hendriks LG, Bas S, van Boxtel MP, Picavet HSJ, et al. Adherence to dietary guidelines and cognitive decline from middle age: the Doetinchem Cohort Study. Am J Clin Nutr. 2021;114(3):871-81.

27 Aartsen MJ, Martin M, Zimprich D. Longitudinal Aging Study A. Gender differences in level and change in cognitive functioning. Results from the Longitudinal Aging Study Amsterdam. Gerontology. 2004;50(1):35-8.

28 van den Kommer TN, Deeg DJH, van der Flier WM, Comijs HC. Time trend in persistent cognitive decline: results from the Longitudinal Aging Study Amsterdam. J Gerontol B Psychol Sci Soc Sci. 2018;73(Suppl 1):S57-64.

29 Miech RA, Breitner JC, Zandi PP, Khachaturian AS, Anthony JC, Mayer L. Incidence of $\mathrm{AD}$ may decline in the early 90 s for men, later for women: the Cache County study. Neurology. 2002;58(2):209-18.

30 Lobo A, Launer LJ, Fratiglioni L, Andersen K, Di Carlo A, Breteler MM, et al. Prevalence of dementia and major subtypes in Europe: a collaborative study of population-based cohorts. Neurologic Diseases in the Elderly Research Group. Neurology. 2000;54(11 Suppl 5):S4-9.

31 Laws KR, Irvine K, Gale TM. Sex differences in cognitive impairment in Alzheimer's disease. World J Psychiatry. 2016;6(1):54-65.

32 Roe CM, Xiong C, Miller JP, Morris JC. Education and Alzheimer disease without dementia: support for the cognitive reserve hypothesis. Neurology. 2007;68(3):223-8.

33 Seshadri S, Beiser A, Kelly-Hayes M, Kase CS, $\mathrm{Au}$ R, Kannel WB, et al. The lifetime risk of stroke: estimates from the Framingham Study. Stroke. 2006;37(2):345-50.

34 Chene G, Beiser A, Au R, Preis SR, Wolf PA, Dufouil C, et al. Gender and incidence of dementia in the Framingham Heart Study from mid-adult life. Alzheimers Dement. 2015; 11(3):310-20. 